\title{
Sleep disturbances in depressed pregnant women and their newborns
}

\author{
Tiffany Field ${ }^{\mathrm{a}, \mathrm{b}, *}$, Miguel Diego $^{\mathrm{a}}$, Maria Hernandez-Reif ${ }^{\mathrm{a}}$, Barbara Figueiredo ${ }^{\mathrm{c}}$, \\ Saul Schanberg ${ }^{\mathrm{d}}$, Cynthia Kuhn ${ }^{\mathrm{d}}$ \\ ${ }^{a}$ Touch Research Institutes, University of Miami School of Medicine, United States \\ ${ }^{\mathrm{b}}$ Fielding Graduate University, CA, United States \\ ${ }^{\mathrm{c}}$ University of Minho, Portugal \\ ${ }^{\mathrm{d}}$ Duke University School of Medicine, NC, United States
}

Received 7 March 2006; received in revised form 31 May 2006; accepted 9 August 2006

\begin{abstract}
Pregnant women $(N=253)$ were recruited during their second trimester of pregnancy $(M=22.3$ weeks gestation $)$ and assigned to depressed $(N=83)$ and non-depressed groups based on a SCID diagnosis of depression. They were then given self-report measures on sleep disturbance, depression, anxiety and anger, and their urine was assayed for norepinephrine and cortisol. These measures were repeated during their third trimester $(M=32.4$ weeks). Their newborns were then observed during sleep. During both the second and third trimesters, the depressed women had more sleep disturbances and higher depression, anxiety and anger scores. They also had higher norepinephrine and cortisol levels. The newborns of the depressed mothers also had more sleep disturbances including less time in deep sleep and more time in indeterminate (disorganized) sleep, and they were more active and cried/fussed more.
\end{abstract}

(C) 2006 Published by Elsevier Inc.

Keywords: Sleep disturbance; Maternal depression; Newborns

Sleep disturbances have been associated with depression in many studies. In a large sample study ( $N=554$ university students), for example, strong correlations were noted between scores on the Center for Epidemiological Studies Depression Scale (CES-D) and the Pittsburgh Sleep Quality Index (PSQI) (De Gennaro, Martina, Curcio, \& Ferrera, 2004). Insomnia is a frequently reported sleep problem in depressed individuals (Agargun, Kara, \& Solmaz, 1997). Others include poor sleep quality, decreased sleep efficiency (or disorganized sleep patterns) increased wakefulness, a greater number of awakenings and more REM sleep on EEG recordings (Saletu-Zyhlarz et al., 2001), as well as longer periods of REM (Wichniak, Antczak, Wierzbicka, \& Jernajczyk, 2002).

In a separate literature focused on sleep problems during pregnancy, similar sleep disturbances have been reported including an increased incidence of insomnia, awakenings, snoring (respiratory problems) and excessive sleepiness (Sahota, Jain, \& Dhand, 2003). These authors suggested that the symptoms were most prevalent during the third trimester. Others have noted these symptoms based on EEG recordings in the women's homes as early as three months gestation, including less deep sleep, more awakening during sleep and lower sleep efficiency (Lee, Zaffke \& McEnany,

\footnotetext{
* Corresponding author. Tel.: +1 3052436781.

E-mail address: tfield@med.miami.edu (T. Field).
} 
2000). In a longitudinal study across pregnancy (from 8 to 38 weeks), a large percentage of the pregnant women experienced sleep disturbances including nightwakings, insomnia and sleep apnea, and these problems remained stable across pregnancy (Mindell \& Jacobson, 2002). In contrast, another longitudinal study reported an increased rate of awakenings across pregnancy (63\% increase in the first trimester and an increase to $80 \%$ by the second trimester) (Lopes et al., 2004).

Thus, the literature is mixed on the stability of sleep problems across pregnancy, and no literature could be found on sleep disturbances in depressed pregnant women.

Potential physiological and biochemical bases for sleep disturbances during pregnancy have been discussed including hormonal and mechanical factors such as changes in respiratory physiology (Santiago, Nolledo, Kinzler, \& Santiago, 2001). However, very little empirical research exists on underlying mechanisms for sleep disturbances, and none could be found for sleep disturbances in depressed pregnant women.

The clinical importance of prenatal sleep disturbance is highlighted by its predictive validity for longer labors and caesarean delivery. In a study using activity recording bracelets during sleep as well as sleep questionnaires, women with disrupted sleep had longer labors and were five times more likely to have caesarean deliveries (Lee \& Gay, 2005). Prenatal sleep disturbances might also be expected to affect fetal activity and neonatal sleep, in as much as fetal activity is significantly elevated in fetuses of depressed women (Dieter et al., 2001), and newborns of depressed mothers are significantly more active and have more disorganized sleep (Jones et al., 1998). The activity and sleep differences might relate to the mother's prenatal sleep disturbances.

The purpose of the current study was to explore: (1) sleep disturbances in depressed pregnant women; (2) relationships between self-reported sleep disturbances and other self-reported problems including depression, anxiety and anger as well as stress indicators including norepinephrine and cortisol levels in pregnant women; (3) the stability of these across pregnancy; (4) relationships between sleep disturbances and fetal activity and neonatal activity and sleep.

\section{Method}

\subsection{Participants}

Two hundred fifty-three pregnant women were recruited during their second trimester of pregnancy $(R=20-24$ weeks; $M=22.3$ weeks) at a prenatal ultrasound clinic. Following informed consent, they were given a diagnostic interview (SCID) to determine whether they were depressed. Thirty-three percent of the sample was depressed $(N=83)$. Twenty percent of the depressed women were diagnosed as having dysthymia, and $80 \%$ as having major depression. They were distributed 55\% Hispanic, 23\% African-American and 22\% non-Hispanic white, they were lower to middle socioeconomic status $(M=4.2$ on the Hollingshead), and their parity averaged 1.2. Their infants averaged 39.2 weeks gestational age and $3143 \mathrm{~g}$ birthweight. The groups did not differ on these characteristics.

\subsection{Procedure}

After the diagnostic interview at the prenatal ultrasound clinic, the women were given self-report measures on sleep disturbances, on depression, and on the co-morbid mood states of anxiety and anger. The pregnant women also provided a urine sample to be assayed for cortisol and norepinephrine. During the ultrasound sessions the activity of their fetuses was recorded. These measures were repeated at the subsequent prenatal visit at 32.4 weeks gestation on an average ( $R=30-35$ weeks). At birth $(M=1.3$ days), the sleep behaviors of their neonates were observed.

\subsection{Self-report measures}

\subsubsection{Structured clinical interview for DSM-IV axis I disorders (SCID)}

The women were given the SCID interview (research version) for the diagnoses of depression and anxiety disorder and to rule out bipolar disorder, schizophrenia and other psychotic disorders. The women were diagnosed with dysthymia or major depression on the SCID based on DSM IV symptoms. The DSM IV defines major depression disorder as five (or more) of the following symptoms being present nearly everyday during the same 2-week period, at least one of the symptoms being depressed mood or loss of interest/pleasure. The symptoms are: (1) depressed most of the day; 
(2) markedly diminished interest or pleasure in all or almost all activities most of the day; (3) significant weight loss when not dieting, or weight gain, or decrease or increase in appetite; (4) insomnia or hypersomnia; (5) psychomotor agitation or retardation; (6) fatigue or loss of energy; (7) feelings of worthlessness or excessive or inappropriate guilt; (8) diminished ability to think or concentrate, or indecisiveness. Dysthymic disorder is defined as depressed mood for most of the day for more days than not for at least 2 years. To be classified as dysthymic, the criteria are the presence of two or more of the following symptoms: (1) poor appetite or overeating; (2) insomnia or hypersomnia; (3) low energy or fatigue; (4) low self-esteem; (5) poor concentration or difficulty making decisions; (6) feelings of hopelessness. Both depression types (major depression and dysthymia) and those diagnosed with co-morbid anxiety disorder were eligible for the study. The SCID was administered by research associates following training and with continuing supervision by a clinical psychologist. In our experience (including a recent survey sample), the majority of the depressed pregnant women who attended the university ultrasound clinic were not taking anti-depressants and were not receiving other treatments for depression.

\subsubsection{Center for epidemiological studies depression scale (CES-D)}

This 20-item scale was included to assess symptoms of depression (Radloff, 1977). The women were asked to report on their feelings during the preceding week. The scale has adequate test/retest reliability ( 0.60 over several weeks), internal consistency (0.80-0.90) and concurrent validity (Wells, Klerman \& Deykin, 1987). A score of 16 on the CES-D is considered the cutpoint for depression (Radloff, 1991).

\subsubsection{State/trait anxiety inventory (STAI)}

This scale was used to assess the co-morbidity of anxiety and depression (Spielberger, Gorsuch \& Lushene, 1970). The state/trait anxiety inventory is comprised of 20 items and is summarized by a score ranging from 20 to 90 and assesses how the person usually feels in terms of severity ("not at all" to "very much so"). Characteristic items include "I feel nervous" and "I feel calm." Research has demonstrated that the STAI has adequate concurrent validity and internal consistency $(r=0.83)$. The cut-off score for high anxiety in our studies has been 48 .

\subsubsection{State/trait anger inventory (STAXI)}

Spielberger et al. (1995) is a 10-item inventory that assesses general feelings of anger based on a 4-point Likert scale ranging from 1 (almost never) to 4 (almost always). Typical questions include "I am quick tempered" and "I fly off the handle". Psychometric properties have been established for the STAXI on diverse ethnic groups. Reliability coefficients have been reported between 0.97 (state) and 0.89 (trait).

\subsubsection{Sleep scale}

Questions on this 15-item scale are rated on a visual analogue anchored at one end with effective sleep responses (e.g., "Did not awaken," "Had no trouble sleeping") and at the opposite end with ineffective sleep responses (e.g., "Was awake 10 h," "Had a lot of trouble falling asleep") (Snyder-Halpern \& Verran, 1987). The participants place a mark across the answer line at the point that best reflects their last night's sleep. A reliability coefficient of 0.82 has been reported for this scale (Snyder-Halpern \& Verran, 1987). The sleep disturbance subscale was used in this study.

\subsubsection{VITAS (VITAS healthcare corporation, 1993)}

The pregnant women completed pain scales, with reference to leg and back pain. Pain perception was rated on a visual analog scale (VAS) ranging from 0 (no pain) to 10 (worst possible pain) and anchored with five faces. The faces, located at two point intervals, ranged from very happy (0), to happy (2); contented (4), somewhat distressed (6), distressed (8), to very distressed (10).

\subsubsection{Urine assays}

Urine samples were collected from the women and frozen. They were subsequently sent to Saul Schanberg, M.D., Ph.D. and Cynthia Kuhn, Ph.D. at Duke University Medical School, where they were assayed for cortisol and norepinephrine as potential biochemical correlates of sleep disturbances. These were done by radioimmunoassays using a specific antiserum from Radioassay Systems Laboratories. The sensitivity of the assays averaged $0.025 \mathrm{ng} /$ tube. The inter-assay and intra-assay coefficients of variation were less than $10 \%$ and $5 \%$ respectively. 


\subsubsection{Fetal activity}

The technician positioned the scanner to obtain a lateral view of the fetus. The observer, who was blind to the mother's group, then watched the fetus for five consecutive minutes. Every $3 \mathrm{~s}$ (a total of $100 \mathrm{samples}$ ), a tape-recorded cue (heard though an earphone) prompted the observer to record fetal activity. Inter-rater reliability, calculated on one-third of the sample for two observers, yielded a Kappa value of 0.92. The percentage of time (\% time) the fetus engaged in movement was calculated.

\subsubsection{Neonatal sleep/wake behavior observation}

A 15-min sleep/wake behavior observation was conducted on the neonates ( $M$ age $=1.3$ days) by researchers who were "blind" to the purpose of the study and to the group assignment of the mothers and infants. Behaviors that occurred during the 15-min period were coded on a laptop computer according to procedures used in the Field et al. study (Field et al., 1986). Prior to the coding, the examiner was trained during four sessions to reach 0.90 reliability. The behavior observation started when the infant was in a quiet, alert state. Interobserver reliability for these averaged 0.84 based on Cohen's Kappa. The observer recorded the infant's predominant state and sleep-wake behaviors using a time-sampling unit methodology with 10-s recording intervals. An adaptation of Thoman's (Thoman, Denenberg, Sievel, Zeidner, \& Becker, 1981) system of sleep recording was used. The sleep state criteria were as follows: (a) quiet sleep (no REM): the infant's eyes are closed and still, and there is no motor activity other than an occasional startle, rhythmic mouthing, or a slight limb movement; (b) active sleep (without REM):the infant's eyes are closed and still, motor activity is present; (c) REM sleep: the infant's eyes are closed, although they may open briefly, rapid eye movements can be detected through closed eyelids, and motor activity may or may not be present; (d) drowsy: the infant's eyes may be opening and closing but have a dull, glazed appearance, motor activity is minimal; (e) inactive alert: the infant is relatively inactive, although there may be occasional limb movement, the eyes are wide open and bright and shiny; (f) active awake: the infant's eyes are open and there is motor activity; (g) crying: the infant's eyes can be open or closed, and motor activity is present as are agitated vocalizations; (h) indeterminate sleep: sleep that is disorganized and does not meet the criteria for other sleep states. In addition to coding behavioral states, the observer also recorded activity.

The total number time-sample units for each behavior was converted to the percent observation time that different states and behaviors occurred for the purpose of data analyses. To ensure reliability of the coding, the observer was first trained to $90 \%$ reliability ( $N$ agreements/ $N$ agreements plus disagreements) prior to the onset of the study. Interobserver reliability was determined during the course of the study by the simultaneous observation and coding of 10 randomly selected sleep observations. The coding was done simultaneously to ensure that the start and end-points were synchronized. The coders used laptop computers and were separated by a post so they could not observe each other's coding. The reliability coefficients were calculated by dividing the number of agreements (same behaviors coded for a given time-sampling unit by both observers) by the number of agreements plus disagreements. The reliability coefficients averaged 0.84 .

\section{Results}

ANOVAs were performed on the sleep disturbance and other self-report variables and urine assays taken during the second and third trimesters for the depressed and non-depressed pregnant women and on the activity levels of their fetuses and on the sleep-wake behaviors of their newborns. As can be seen in Table 1, during the second trimester, the depressed women: (1) had higher scores on the self-report measures including sleep disturbances, depression, anxiety and anger; (2) higher norepinephrine and cortisol levels.

As can be seen in Table 2, during the third trimester, the depressed women had higher scores again on the sleep disturbances, depression, anxiety and anger self-report scales.

Pearson correlation analyses were conducted on the variables measured during the second and third trimesters. As can be seen in Table 3, sleep disturbances, as reported during the second trimester $(M=22.3$ weeks gestation), were significantly correlated with the following variables during the same trimester including: (1) depression (CES-D), (2) anxiety (STAI); (3) norepinephrine (NE) and, (4) fetal movement observed during the ultrasound session. Sleep disturbances as reported during the second trimester were significantly correlated with the following variables collected during the third trimester ( $M=32.4$ weeks gestation) including: (1) sleep disturbances; (2) anxiety (STAI); (3) anger (STAXI); (4) cortisol. 
Table 1

Means for sleep disturbances and other variables taken during the second trimester for depressed and non-depressed pregnant women

\begin{tabular}{|c|c|c|c|c|}
\hline \\
\hline \multicolumn{5}{|l|}{$\frac{\text { Groups }}{\text { Variables }}$} \\
\hline Sleep disturbances & $39.9(20.9)$ & $46.1(21.2)$ & 4.9 & 0.03 \\
\hline Depression (CES-D) & $9.0(3.9)$ & $24.7(7.6)$ & 36.1 & 0.000 \\
\hline Anxiety (STAI) & $32.6(8.4)$ & $40.2(15.1)$ & 68.5 & 0.000 \\
\hline Anger (STAXI) & $17.7(6.7)$ & $25.1(11.5)$ & 117.7 & 0.000 \\
\hline Norepinephrine (NE) & $54.9(22.7)$ & $61.6(28.6)$ & 4.7 & 0.05 \\
\hline Cortisol (CO) & $137.1(86.4)$ & $154.8(89.4)$ & 4.8 & 0.05 \\
\hline
\end{tabular}

Standard deviations in parentheses.

Table 2

Means for sleep disturbance and other variables taken during the third trimester for depressed and non-depressed pregnant women

Groups

\begin{tabular}{|c|c|c|c|c|}
\hline Variables & Non-depressed & Depressed & $F$ & $p$ \\
\hline Sleep disturbances & $41.2(21.6)$ & $48.7(23.1)$ & 5.8 & 0.05 \\
\hline Depression (CES-D) & $9.5(5.5)$ & $21.9(9.7)$ & 56.4 & 0.000 \\
\hline Anxiety (STAI) & $33.8(8.4)$ & $43.7(10.8)$ & 53.7 & 0.000 \\
\hline Anger (STAXI) & $15.9(3.9)$ & $21.6(9.0)$ & 25.2 & 0.000 \\
\hline
\end{tabular}

Standard deviations in parentheses.

Table 3

Significant correlations between sleep disturbances as reported during the second trimester and sleep disturbances and other variables as reported by pregnant women during their second and third trimesters

\begin{tabular}{llll}
\hline \multicolumn{2}{l}{ Sleep disturbances at second trimester } & \multicolumn{3}{l}{} \\
\hline Second trimester variables and coefficients & & \multicolumn{2}{l}{ Third trimester variables and coefficients } \\
Depression (CESD) & $0.23^{\mathrm{a}}$ & Sleep disturbance & $0.52^{\mathrm{b}}$ \\
Anxiety (STAI) & $0.30^{\mathrm{b}}$ & Anxiety (STAI) & $0.36^{\mathrm{b}}$ \\
Norepinephrine (NE) & $0.36^{\mathrm{b}}$ & Anger (STAXI) & $0.25^{\mathrm{b}}$ \\
Fetal activity & $0.25^{\mathrm{a}}$ & Cortisol (Co) & $0.28^{\mathrm{a}}$ \\
\hline
\end{tabular}

a $p<0.05$.

b $p<0.01$.

As can be seen in Table 4, sleep disturbances as reported during the third trimester ( $M=32.4$ week gestation) were significantly related to second trimester variables including: (1) sleep disturbances; (2) depression; (3) anxiety; (4) anger; (5) cortisol. Sleep disturbances as reported during the third trimester were also significantly correlated with variables from the same trimester including: (1) anxiety; (2) back pain; (3) leg pain.

Table 4

Significant correlations between sleep disturbances as reported during the third trimester and sleep disturbances and other variables in depressed pregnant women at their second and third trimesters

\begin{tabular}{|c|c|c|c|}
\hline \multicolumn{4}{|c|}{ Sleep disturbances at third trimester } \\
\hline \multicolumn{2}{|c|}{ Second trimester variables and coefficients } & \multicolumn{2}{|c|}{ Third trimester variables and coefficients } \\
\hline Sleep disturbances & $0.52^{b}$ & Anxiety (STAI) & $0.37^{\mathrm{b}}$ \\
\hline Depression (CESA) & $0.30^{\mathrm{b}}$ & Back pain & $0.38^{\mathrm{b}}$ \\
\hline Anxiety (STAI) & $0.26^{\mathrm{a}}$ & Leg pain & $0.41^{b}$ \\
\hline Anger (STAXI) & $0.22^{\mathrm{a}}$ & & \\
\hline Cortisol & $0.25^{\mathrm{a}}$ & & \\
\hline
\end{tabular}

\footnotetext{
${ }^{\text {a }} p<0.05$.
}

b $p<0.01$. 
Table 5

Means for sleep measures for newborns of depressed and non-depressed mothers

\begin{tabular}{|c|c|c|c|c|}
\hline \multicolumn{5}{|l|}{ Groups } \\
\hline Variables & Non-depressed & Depressed & $F$ & $p$ \\
\hline Deep sleep & $48.2(35.4)$ & $32.7(35.9)$ & 6.7 & 0.01 \\
\hline Active sleep & $19.7(20.6)$ & $17.4(18.2)$ & & N.S \\
\hline Drowsiness & $9.8(10.2)$ & $14.3(15.3)$ & & N.S \\
\hline Quiet alert & $12.0(10.0)$ & $12.2(10.3)$ & & N.S \\
\hline Active alert & $5.1(11.9)$ & $5.9(11.8)$ & & N.S \\
\hline Fussing/crying & $3.0(8.3)$ & $7.1(6.7)$ & 4.1 & 0.05 \\
\hline Indeterminate sleep & $2.1(5.1)$ & $9.8(19.8)$ & 9.0 & 0.003 \\
\hline No activity & $60.9(24.9)$ & $49.2(30.2)$ & 6.1 & 0.02 \\
\hline
\end{tabular}

Standard deviations in parentheses. Percent time sleep behaviors/states occurred.

As can be seen in Table 5, the newborns of depressed mothers spent: (1) a lesser percent of the sleep/wake observation time in deep sleep and, (2) a greater percent of the sleep time in a fussing/crying state, in indeterminate sleep, and being active (or less time showing no activity).

\section{Discussion}

Although no literature could be found on sleep disturbances in depressed pregnant women, the findings of this study were not surprising, given the frequently reported relationships between sleep disturbances and pregnancy (Lee et al., 2000; Sahota et al., 2003) and between sleep disturbances and depression (De Gennaro et al., 2004; Saletu-Zyhlarz et al., 2001).

The stability of sleep disturbances across the second and third trimester is consistent with other findings on sleep disturbances in non-depressed pregnant women (Mindell \& Jacobson, 2002). Although hormonal and mechanical factors have been associated with sleep disturbances (Santiago et al., 2001), very little is known about contributing or associated factors. The depressed pregnant women in this study not only suffered from sleep disturbances, but also experienced problems that are often co-morbid with depression including high anxiety and anger, and higher stress levels suggested by their elevated norepinephrine and cortisol levels. These problems, in themselves, may have contributed to the sleep disturbances, or at least compounded the effects of depression on sleep disturbances.

The sleep disturbances, were also related to leg and back pain. Pain syndromes have been known to result from sleep disturbances and the associated release of pain-causing chemicals such as Substance P in fibromyalgia (Field et al., 2002). The increased fetal activity and sleep disturbances could also be a reciprocal process, with an active fetus interrupting sleep, or disruptive sleep increasing fetal activity.

Of significant concern were the apparent effects of the women's depression and sleep disturbances on the newborn's sleep including spending less time in deep sleep and more time in indeterminate (disorganized) sleep and fussing/crying. The increased neonatal activity may have followed from increased fetal activity, as has been reported before for fetuses and neonates of depressed women (Diego et al., 2002; Dieter et al., 2001; Field et al., 2004a).

How elevated fetal activity and neonatal sleep disturbances are related to later development is unknown and requires further study. These problems, however, highlight the need for early interventions to decrease sleep disturbances in depressed pregnant women. Pregnancy massage, for example, has been noted to reduce prenatal depression in at least two studies (Field, Diego, Hernandez-Reif, Schanberg,\& Kuhn 2004), and to decrease sleep disturbances in newborns born to depressed women (Field, Hernandez,\& Diego, 2006).

\section{Acknowledgements}

We would like to thank the parents and infants who participated in this study. This research was supported by a merit award (MH\# 46586) and Senior Research Scientist Awards (MH\# 00331 and AT\# 001585) and a March of Dimes Grant (\#12-FYO3-48) to Tiffany Field and funding from Johnson \& Johnson Pediatric Institute to the Touch Research Institutes. Correspondence and requests for reprints should be sent to Tiffany Field, Ph.D., Touch Research Institutes, 
University of Miami School of Medicine, P.O. Box 016820, Miami, Florida, 33101. Business phone number (305) 243-6781.

\section{References}

Agargun, M. Y., Kara, H., \& Solmaz, M. (1997). Sleep disturbances and suicidal behavior in patients with major depression. Journal of Clinical Psychiatry, 58, 249-251.

De Gennaro, L., Martina, M., Curcio, G., \& Ferrera, M. (2004). The relationship between alexithmia, depression, and sleep complaints. Psychiatry Research, 128, 253-258.

Diego, M., Dieter, J., Field, T., LeCanuet, J., Hernandez-Reif, M., Beutler, J., et al. (2002). Fetal activity following vibratory stimulation of the mother's abdomen and foot and hand massage. Developmental Psychobiology, 41, 396-406.

Dieter, J., Field, T., Hernandez-Reif, M., Jones, N. A., Lecanuet, J. P., Salman, F. A., et al. (2001). Maternal depression and increased fetal activity. Journal of Obstetrics and Gynaecology, 21, 468-473.

Field, T., Diego, M., Cullen, C., Hernandez-Reif, M., Sunshine, W., \& Douglas, S. (2002). Fibromyalgia pain and substance P decrease and sleep improves after massage therapy. Journal of Clinical Rheumatology, 8, 72-76.

Field, T., Diego, M., Dieter, J., Hernandez-Reif, M., Schanberg, S., Kuhn, C., et al. (2004). Prenatal depression effects on the fetus and neonate. Infant Behavior \& Development, 27, 216-229.

Field, T., Diego, M., Hernandez-Reif, M., Schanberg, S., \& Kuhn, C. (2004). Massage therapy effects on depressed pregnant women. Journal of Psychosomatic Obstetrics \& Gynecology, 25(2), 115-122.

Field, T., Hernandez-Reif, M., \& Diego, M. (2006). Newborns of depressed mothers who received moderate versus light pressure massage during pregnancy. Infant Behavior and development, 29, 54-58.

Field, T., Hernandez-Reif, M., Hart, S., Theakston, H., Schanberg, S., Kuhn, C., et al. (1999). Pregnant women benefit from massage therapy. Journal of Psychosomatic Obstetrics \& Gynecology, 20, 31-38.

Field, T., Schanberg, S. M., Scafidi, F., Bauer, C. R., Vega-Lahr, N., Garcia, R., et al. (1986). Tactile/kinesthetic stimulation effects on preterm neonates. Pediatrics, 77, 654-658.

Jones, N. A., Field, T., Fox, N. A., Davalos, M., Lundy, B., \& Hart, S. (1998). Newborns of mothers with depressive symptoms are physiologically less developed. Infant Behavior and Development, 21, 537-541.

Lee, K. A., \& Gay, C. L. (2005). Sleep in late pregnancy predicts length of labor and type of delivery. American Journal of Obstetric and Gynecology, 191, 2041-2046.

Lee, K. A., Zaffke, M. E., \& McEnany, G. (2000). Parity and sleep patterns during and after pregnancy. Obstetric and Gynecology, 95, 14-18.

Lopes, E. A., Carvalho, L. B., Seguro, P. B., Mattar, R., Silva, A. B., Prado, L. B., et al. (2004). Sleep disorders in pregnancy. Arquivos de Neuro-Psiquiatria, 62, 217-221.

Mindell, J. A., \& Jacabson, B. J. (2000). Sleep disturbances during pregnancy. Journal of Obstetrics and Gynecology of Neonatal Nursing, 29, 590-597.

Radloff, L. S. (1977). The CES-D Scale: A self-report depression scale for research in the general population. Journal of Applied Psychological Measures, 1, 385-401.

Radloff, L. (1991). The use of the Center for Epidemiological Studies Depression Scale in adolescents and young adults. Journal of Youth and Adolescence, 20, 149-165.

Sahota, P. K., Jain, S. S., \& Dhand, R. (2003). Sleep disorders in pregnancy. Current Opinion Pulmonary Medicine, 9, $477-483$.

Saletu-Zyhlarz, G. M., Abu-Bakr, M. H., Anderer, P., Semler, B., Decker, K., Parapatic, S., et al. (2001). Insomnia related to dysthymia: Polysomnographic and psychometric comparison with normal controls and acute therapeutic trails with trazodone. Neuropsychobiology, 44, $139-149$.

Santiago, J. R., Nolledo, M. S., Kinzler, W., \& Santiago, T. V. (2001). Sleep and sleep disorders in pregnancy. Annales International Medicine, 134, 396-408.

Snyder-Halpern, R., \& Verran, J. A. (1987). Instrumentation to describe subjective sleep characteristics in healthy subjects. Research in Nursing Health, 10, 155-163.

Spielberger, C., Gorsuch, R. L., \& Lushene, R. E. (1970). The state-trait anxiety inventory. Palo Alto, CA: Consulting Psychologists Press.

Spielberger, C. D., Ritterband, L. M., Sydeman, S. J., Reheiser, E. C., \& Unger, K. K. (1995). Assessment of emotional states and personality traits: Measuring psychological vital signs. In Clinical personality assessment: Practical approaches. New York: Oxford University Press.

Thoman, E. B., Denenberg, V. H., Sievel, J., Zeidner, L. P., \& Becker, P. (1981). State organization in neonates: Developmental inconsistency indicates risk for developmental dysfunction. Neuropediatrics, 12, 45-54.

Wells, V. E., Klerman, G. L., \& Deykin, E. Y. (1987). The prevalence of depressive symptoms in college students. Social Psychiatry, 22, 20-28. 